\title{
MHD Mixed Convection Flow of A Micropolar Fluids Through Porous Medium Towards A Stagnation Point on A Vertical Surface
}

\author{
A. Haritha, Y. Devasena \\ School of Engineering and Technology, SPMVV, Tirupati (A.P)
}

\begin{abstract}
The steady mixed convection flow of a viscous incompressible micropolar fluid through porous medium towards a stagnation point over a vertical surface when the buoyancy force assists and opposes the flow has been investigated. The governing nonlinear partial differential equations are transformed into a system of ordinary differential equations and then solved by employing Runge-Kutta fourth order method along with shooting technique. The velocity, microrotation and temperature profiles for different governing parameters are illustrated. It has been observed that with the increase of material parameter $K$ for assisting flow and opposing flow the velocity and angular velocity decreases whereas the temperature increases. The presence of porous parameter K1 enhances the velocity and angular velocity but it decreases the temperature for assisting and opposing flows. Numerical results for skin - friction coefficient, the couple wall stress and the local Nusselt number are discussed.
\end{abstract}

Keywords: Mixed convection, Micropolar fluid, Magnetohydrodynamic flows, Porous medium.

\section{Introduction}

The theory of micropolar fluids has risen from the need to model many engineering processes involving non-Newtonian fluids containing micro-constituents such as blood flow, lubricants, colloidal fluids, liquid crystals that cannot described by the classical Newtonian fluid. The model of micropolar fluids primarily introduced by C. A. Eringen [1] taking into account the internal characteristics of subtractive particles, which are allowed to undergo rotation. In the micropolar fluid theory, apart from the classical velocity field, the microrotation vector and the gyration parameter are introduced to investigate the kinematics of microrotations and provides a good mathematical model for the non-Newtonian behavior. A good list of references on the published papers for this fluid can be found in Eringen [2] and Ishak et al. [3]. Gorla [4] studied mixed convection in a micropolar fluid from a vertical surface with uniform heat flux. Anuar Ishak et al. [5] analyzed MHD flow of a micropolar fluid towards a stagnation point on a vertical surface.

The flow through a porous channel with expanding or contracting walls has gained great importance because of its applications in biophysical flows. For example, the model of pulsating diaphragms, filtration, blood flow and artificial dialysis, binary gas diffusion, the model of air and blood circulation in the respiratory system. In recent years, many authors have studied

unsteady free convection flow of a micropolar fluid with or without a magnetic field through a porous medium. For example, Srinivasacharya et al. [6] discussed the effects of microrotation and frequency parameters on an unsteady flow of micropolar fluid between two parallel porous plates with a periodic suction. Bhargara et al. [7] obtained a numerical solution of a free convection MHD micropolar fluid flow between two parallel porous vertical plates by means of the quasi-linearization method. Rahman and Sattar [8] studied magnetohydrodynamic convective flow of a micropolar fluid past a vertical porous plate in the presence of heat generati8ion/absorption.

The present paper investigates MHD mixed convection flow of micropolar fluids through porous medium towards a stagnation point on a vertical surface. Using the similarity transformations, the governing equations have been transformed into a system of ordinary differential equations and then solved by employing Runge-Kutta fourth order method along with shooting technique. The results for velocity, microrotation and temperature functions are carried out for different parameters. The skin friction, the couple wall stress and the rate of heat transfer have been computed.

\section{Mathematical Analysis}

Consider the steady, two-dimensional flow of an incompressible electrically conducting micropolar fluid through porous medium near the stagnation point on a vertical heated plate. It is assumed that the velocity of the flow external to the boundary layer $\mathrm{U}(\mathrm{x})$ and the temperature $\mathrm{T}_{\mathrm{w}}(\mathrm{x})$ of the plate are proportional to the distance $\mathrm{x}$ from the stagnation point,i.e. $\mathrm{U}(\mathrm{x})=\mathrm{ax}$ and $\mathrm{T}_{\mathrm{w}}(\mathrm{x})=\mathrm{T}_{\infty}+\mathrm{bx}$, where $\mathrm{a}$ and $\mathrm{b}$ are constants. $\mathrm{A}$ uniform magnetic field of strength $\mathrm{B}_{0}$ is assumed to be applied in the positive y-direction normal to the plate. The 
magnetic Reynolds number of the flow is taken to be small so that the induced magnetic field is negligible. Under these assumptions along with the Boussinesq and boundary layer approximations, the system of equations, which models the flow is given by

$$
\begin{aligned}
& \frac{\partial u}{\partial x}+\frac{\partial v}{\partial y}=0 \\
& u \frac{\partial u}{\partial x}+v \frac{\partial u}{\partial y}=U \frac{d u}{d x}+\left(\frac{\mu+k}{\rho}\right) \frac{\partial^{2} u}{\partial y^{2}}+\frac{k}{\rho} \frac{\partial N}{\partial y}-\frac{v}{K_{0}} u+\frac{\sigma B_{0}^{2} u}{\rho}(U-u)+g \beta\left(T-T_{\infty}\right) \\
& \rho j\left(u \frac{\partial N}{\partial x}+v \frac{\partial N}{\partial y}\right)=\gamma \frac{\partial^{2} N}{\partial y^{2}}-k\left(2 N+\frac{\partial u}{\partial y}\right) \\
& u \frac{\partial T}{\partial x}+v \frac{\partial T}{\partial y}=\alpha \frac{\partial^{2} T}{\partial y^{2}}
\end{aligned}
$$

subject to the boundary conditions

$$
\begin{aligned}
& \mathrm{u}=0, \quad \mathrm{v}=0, \quad \mathrm{~N}=-\frac{1}{2} \frac{\partial u}{\partial y}, \quad T=T_{w}(x) \text { at } y=0 \\
& \mathrm{u} \rightarrow U(x), \quad N \rightarrow 0, T \rightarrow T_{\infty} \quad \text { as } y \rightarrow \infty
\end{aligned}
$$

where $\mathrm{u}$ and $\mathrm{v}$ are the velocity components in the $\mathrm{x}$ - and $\mathrm{y}$-directions, respectively, $\mathrm{T}$ is the fluid temperature in the boundary layer, $\mathrm{N}$ is the component of the microrotation vector normal to the $\mathrm{x}-\mathrm{y}$ plane, $\mu$ is the dynamic viscosity, $\mathrm{k}$ is the microrotation viscosity, $\beta$ is the thermal expansion coefficient, $v$ is the kinematic viscosity coefficient, $\mathrm{K}_{0}$ is the permeability of the medium, $\rho$ is the fluid density, $\mathrm{j}$ is the microinertia density, $\alpha$ is the thermal diffusivity and $\gamma$ is the spin gradient viscosity by assuming that $\gamma=(\mu+\mathrm{k} / 2) \mathrm{j}=$ $\mu(1+K / 2)$ j, where $K=k / \mu$ is the material parameter.

The continuity equation is satisfied by the Cauchy Reimann equations

$$
\mathrm{u}=\frac{\partial \Psi}{\partial y}, \quad \mathrm{v}=\frac{\partial \Psi}{\partial x}
$$

In order to transform equations (2), (3) and (4) into a set of ordinary differential equations, the following similarity transformations and dimensionless variables are introduced:

$$
\eta=\left(\frac{a}{v}\right)^{1 / 2} y, \quad f(\eta)=\frac{\psi}{(a v)^{1 / 2} x}, \quad h(\eta)=\frac{N}{a(a / v)^{1 / 2} x}, \quad \theta(\eta)=\frac{T-T_{\infty}}{T_{w}-T_{\infty}}
$$

The transformed ordinary differential equations are

$$
\begin{aligned}
& (1+\mathrm{K}) f^{\prime \prime \prime}+f f^{\prime \prime}+1-f^{2}+K h^{\prime}+M-\left(M+\frac{1}{K_{1}}\right) f^{\prime}+\lambda \theta=0 \\
& \left(1+\frac{K}{2}\right) h^{\prime \prime}+f h^{\prime}-f^{\prime} h-K\left(2 h+f^{\prime \prime}\right)=0 \\
& \frac{1}{P r} \theta^{\prime \prime}+f \theta^{\prime}-f^{\prime} \theta=0
\end{aligned}
$$

The boundary conditions then become

$f(0)=0, f^{\prime}(0)=0, \quad h(0)=-\frac{1}{2} f^{\prime \prime}(0), \quad \theta(0)=1$,

$f^{\prime}(\eta) \rightarrow 1, \quad h(\eta) \rightarrow 0, \quad \theta(\eta) \rightarrow 0$ as $\eta \rightarrow \infty$

Where $\mathrm{j}=v / \mathrm{a}$ is a characteristic length, $\mathrm{M}=B_{0}^{2} \sigma /(\rho a)$ is the magnetic parameter, $\lambda=G r_{x} / \operatorname{Re}_{x}^{2}$ is the buoyancy parameter, $G r_{x}=g \beta\left(T_{w}-T_{\infty}\right) x^{3} / v^{2}$ is the local Grashof number, $R e_{x}=U x / v$ is the local Reynolds number, $K_{1}=K_{0} a / v$ is permeability parameter and $\operatorname{Pr}=v / \alpha$ is the Prandtl number. In the above equations, primes denotes the differentiation with respect to $\eta$, here $\lambda$ is a constant with $\lambda<0$ and $\lambda>0$ correspond to the opposing and assisting flows respectively while $\lambda=0$ is for pure forced convection flow. 
The physical quantities the skin friction coefficient $\mathrm{C}_{\mathrm{f}}$, the local Nusselt number $\mathrm{Nu}_{\mathrm{x}}$ are defined as

$$
\mathrm{C}_{\mathrm{f}}=\frac{T_{w}}{\rho U^{2} / 2}, \quad \quad \mathrm{Nu}_{\mathrm{x}}=\frac{x q_{w}}{K\left(T_{w}-T_{\infty}\right)},
$$

Where the wall shear stress $T_{w}$ and the heat flux $q_{w}$ are given as

$$
T_{w}=\left[(\mu+k) \frac{\partial u}{\partial y}+k N\right]_{y=0}, \quad q_{w}=-k\left(\frac{\partial T}{\partial y}\right)_{y=0},
$$

With $\mathrm{k}$ being the thermal conductivity, using the similarity variables we obtain,

$$
\frac{1}{2} C_{f} R e_{x}^{1 / 2}=\left(1+\frac{K}{2}\right) f^{\prime \prime}(0), \quad N u_{x} / R e_{x}^{\frac{1}{2}}=-\theta^{\prime}(0)
$$

\section{Results and Discussions}

The numerical results for the velocity, angular velocity and temperature are obtained for different values of the parameters encountered in the problem. Physically $\lambda>0$ means heating of the fluid or cooling of the surface (assisting flow), $\lambda<0$ cooling of the fluid or heating of the surface (opposing flow) and $\lambda=0$ means the forced convection flow.

Figs. 1-3 represents the axial velocity, microrotation and temperature distribution with $\eta$ for the different values of material parameter $\mathrm{K}$ in the case of assisting and opposing flows. It is observed that both the axial velocity and angular velocity decrease in case of assisting and opposing flows with increase of material parameter $\mathrm{K}$ while the temperature increases in both cases. The axial velocity, angular velocity and temperature profiles in case of assisting and opposing flows with different values of Prandtl number Pr are seen in figs. 4-6. It is found that both axial velocity and angular velocity decreases when $\lambda$ is positive while increase in case of opposing flow i.e, $\lambda$ is negative with the increase of Pr and the temperature decreases in both cases. Figs. 7-9 show the plots of velocity, angular velocity and temperature for different values of magnetic parameter M. It is seen that both axial velocity and angular velocity increases with increase of $M$ in assisting and opposing flows while temperature decreases in both cases. Figs.10-12 represents axial velocity, angular velocity and temperature for different values of porous parameter $\mathrm{K}_{1}$ in the case of assisting and opposing flows. It is observed that with increase of porous parameter $\mathrm{K}_{1}$ the velocity and angular velocity increases in both assisting and opposing flows but the temperature decreases. The effect of velocity and angular velocity for different values of buoyancy parameter $\lambda$ are shown in Figs. 13-14. It is seen that velocity increases and angular velocity decreases with increase of buoyancy parameter.

Figs. 15-18 demonstrates the local skin friction coefficient $f^{\prime \prime}(0)$ and the local Nusselt number $-\theta^{\prime}(0)$ with different variations. Fig. 15 shows that the skin friction coefficient increase for increasing values of both $\mathrm{k} 1$ and $\lambda$ where as an opposite behavior in Nusselt number (Fig. 16). Fig. 17 depicts that skin friction coefficient

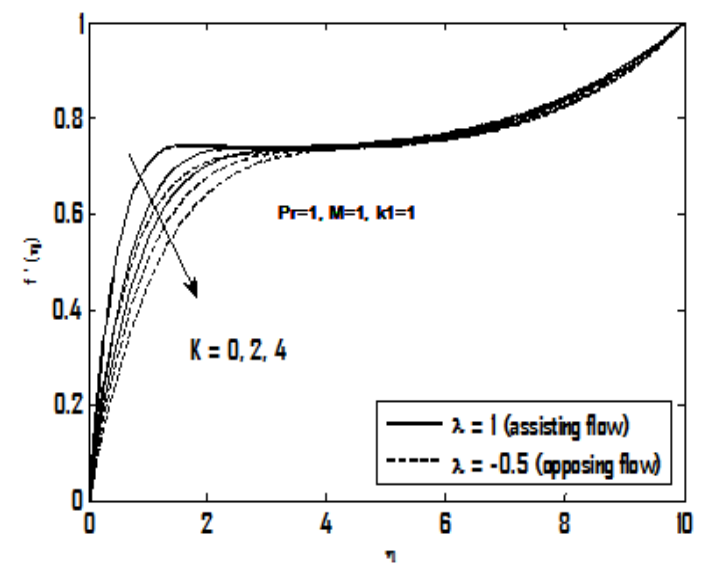

Fig.(1) Velocity profiles for different values of $K$ and $\lambda$

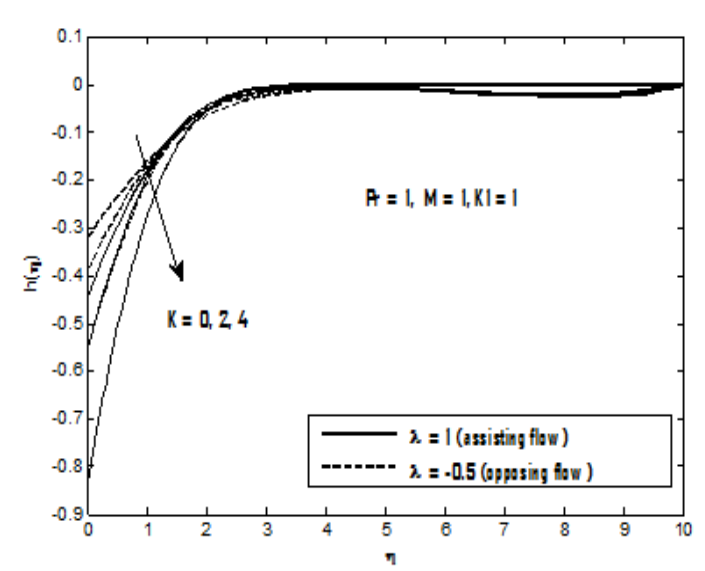

Fig.(2) Angular velocity for different values of $K$ and $\lambda$ 

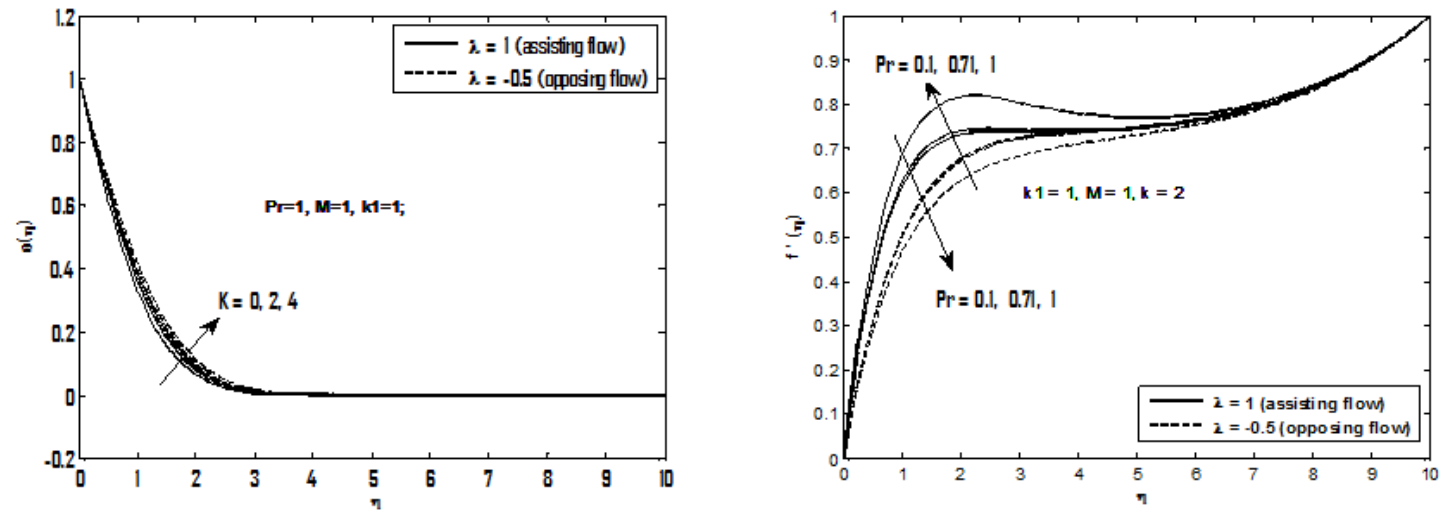

Fig.(3) Temperature profiles for different values of $\mathrm{K}$ and $\lambda$ Fig. (4) Velocity profiles for different values of $\operatorname{Pr}$ and $\lambda$
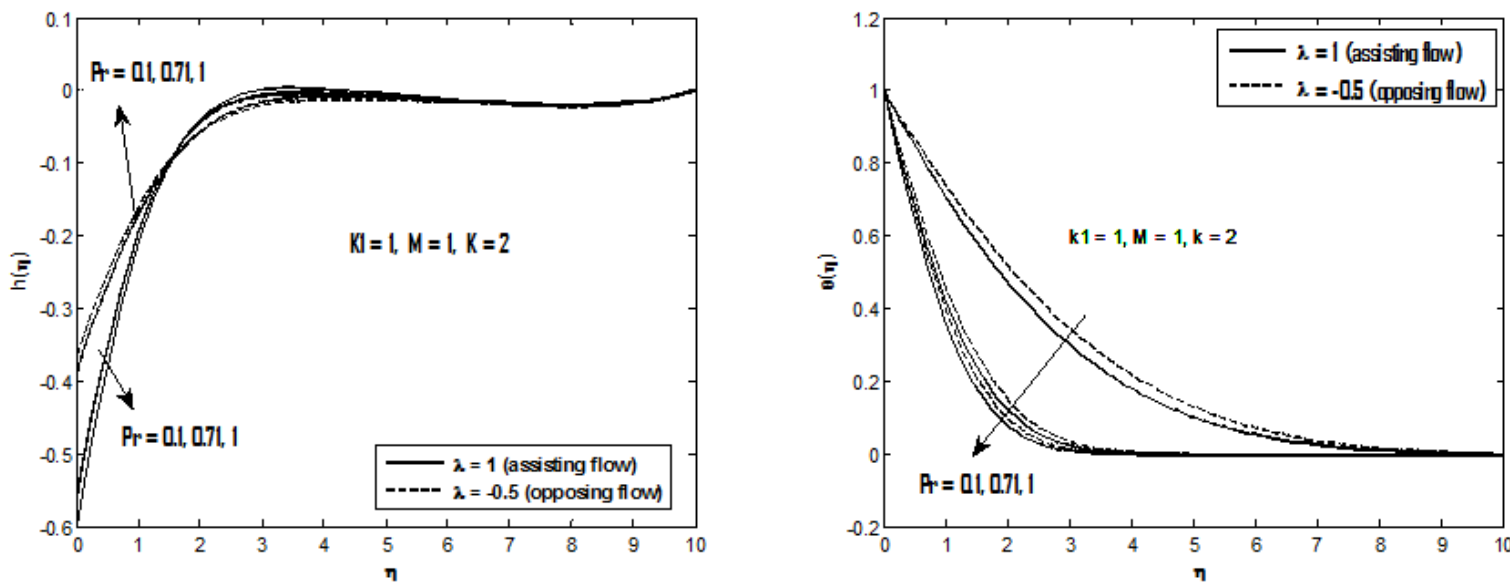

Fig.(5) Angular velocity for different values of $\operatorname{Pr}$ and $\lambda$

Fig.(6) Temperature profiles for different values of $\operatorname{Pr}$ and $\lambda$
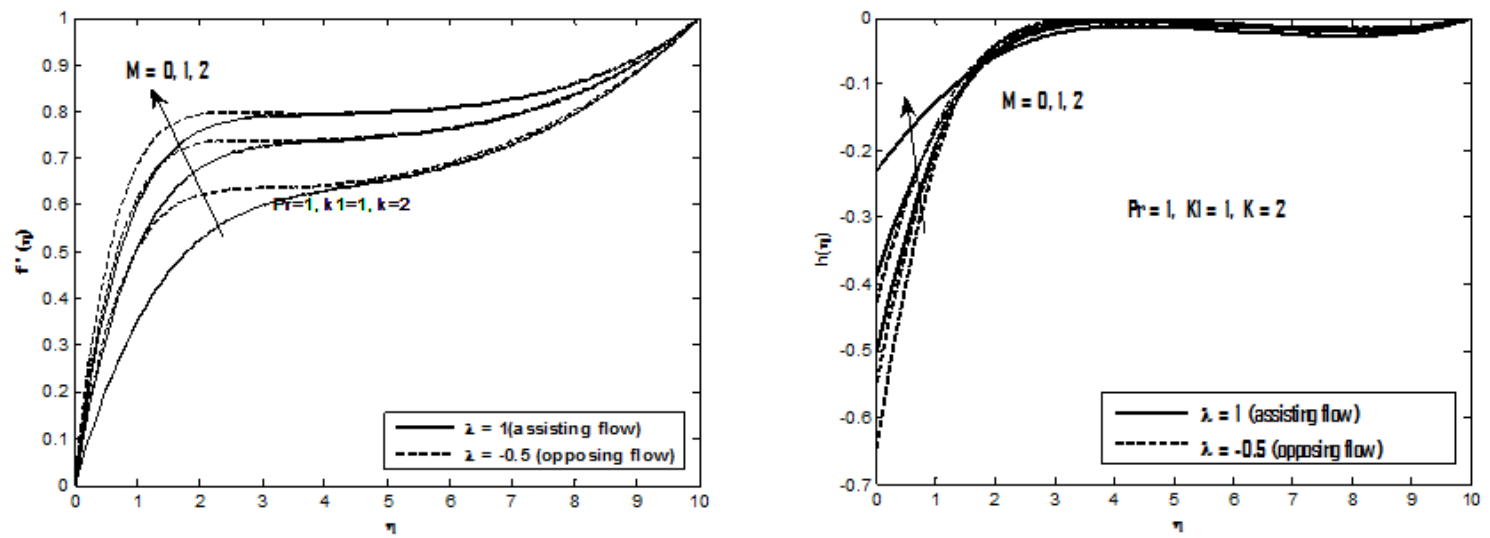

Fig. (7) Velocity profiles for different values of $M$ and $\lambda$ Fig.(8) Angular Velocity profiles for different values of $M$ and $\lambda$
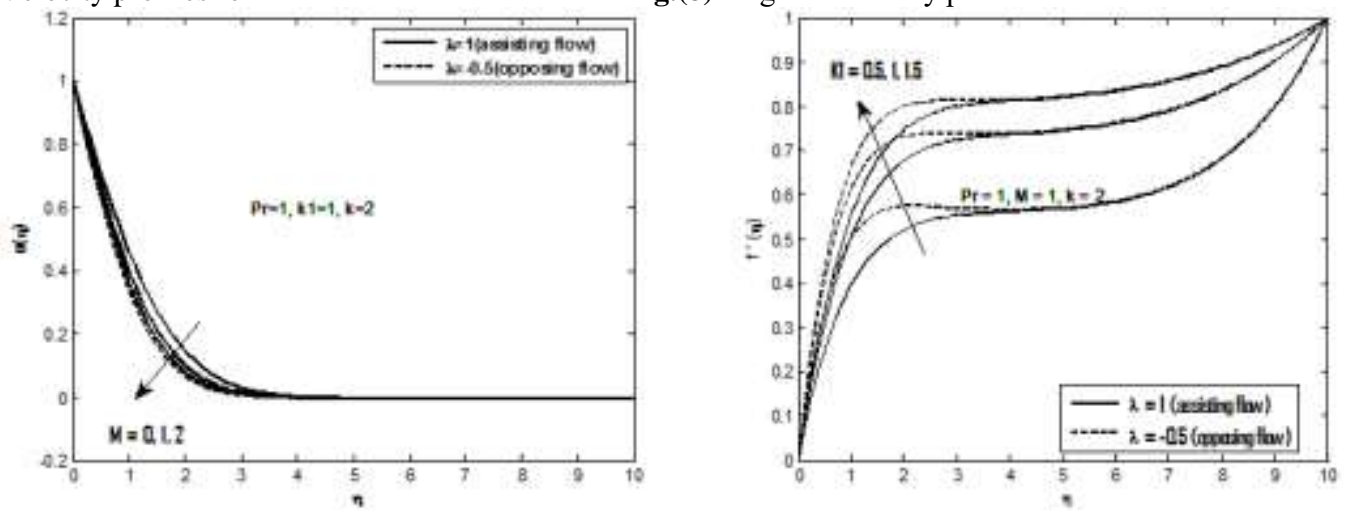

Fig.(9) Temperature profiles for different values of $M$ and $\lambda$

Fig.(10) Velocity profiles for different values of $\mathrm{K} 1$ and $\lambda$ 


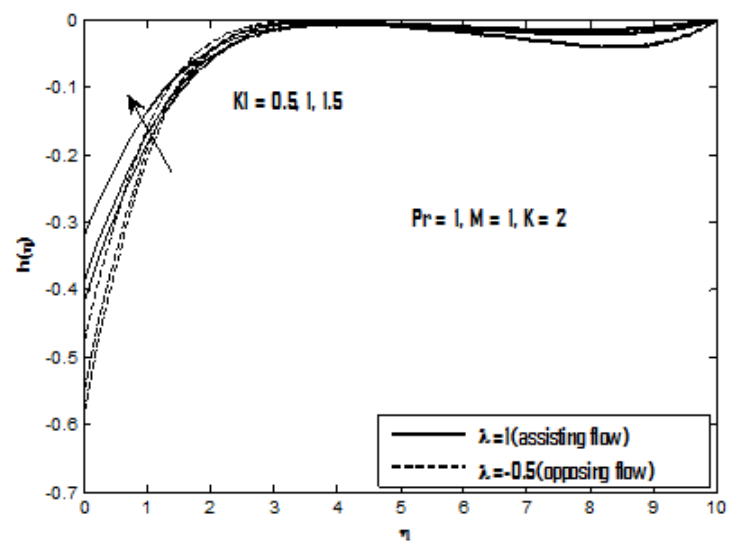

Fig.(11) Angular Velocity profiles for different values of $\mathrm{K} 1$ and $\lambda$

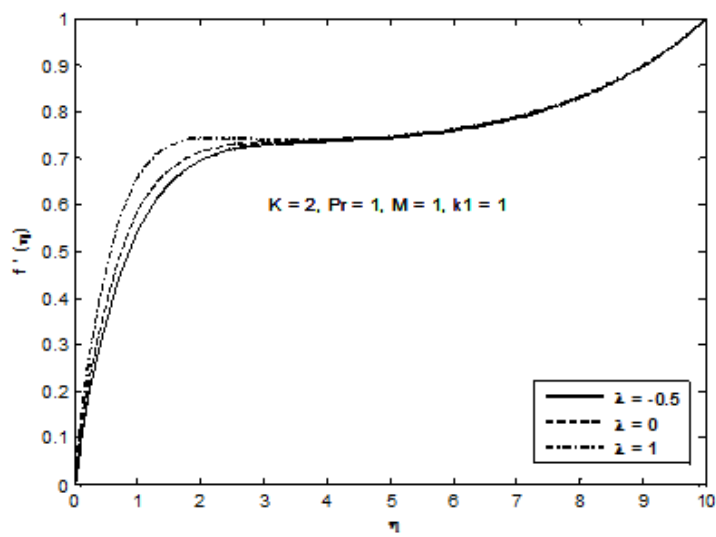

Fig. (13) Velocity profiles for different values of $\lambda$

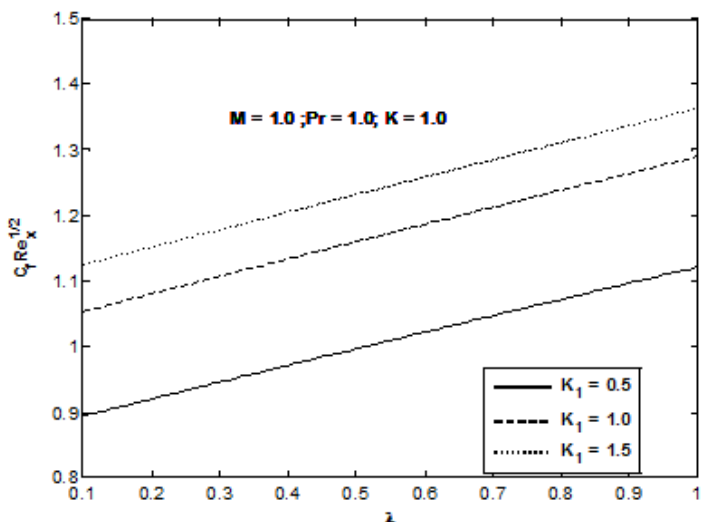

Fig (15) Variation of skin friction coefft. $f^{\prime \prime}(0)$ with $\lambda$ and $K_{1}$. Fig (16) Variation of local Nusselt no. $-\theta^{\prime}(0)$ with $\lambda$ and $K_{1}$

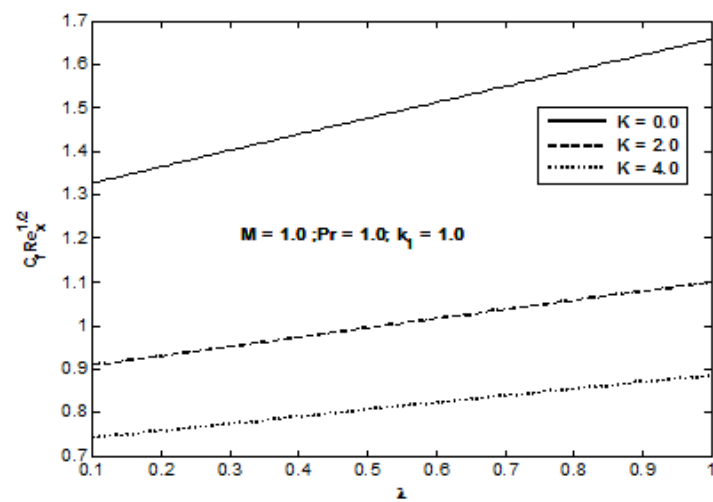

Fig. (17) Variation of skin friction coefft. $f^{\prime \prime}(0)$ with $\lambda$ and K. Fig (18) Variation of local Nusselt no. $-\theta^{\prime}(0)$ with $\lambda$ and K. DOI: $10.9790 / 5728-1204043237$

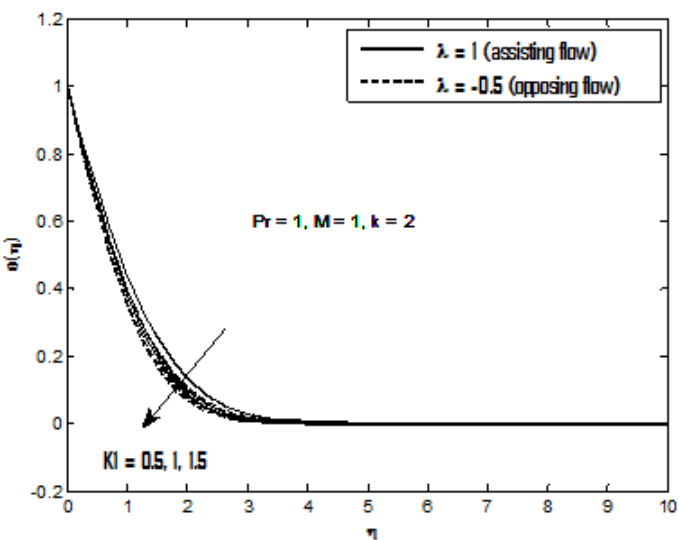

Fig. (12) Temperature for different values of $\mathrm{K} 1$ and $\lambda$

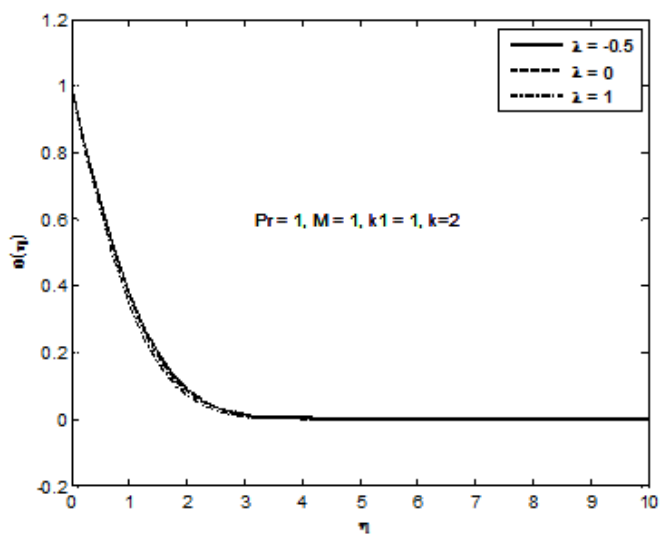

Fig. (14) Angular Velocity profiles for different values of $\lambda$
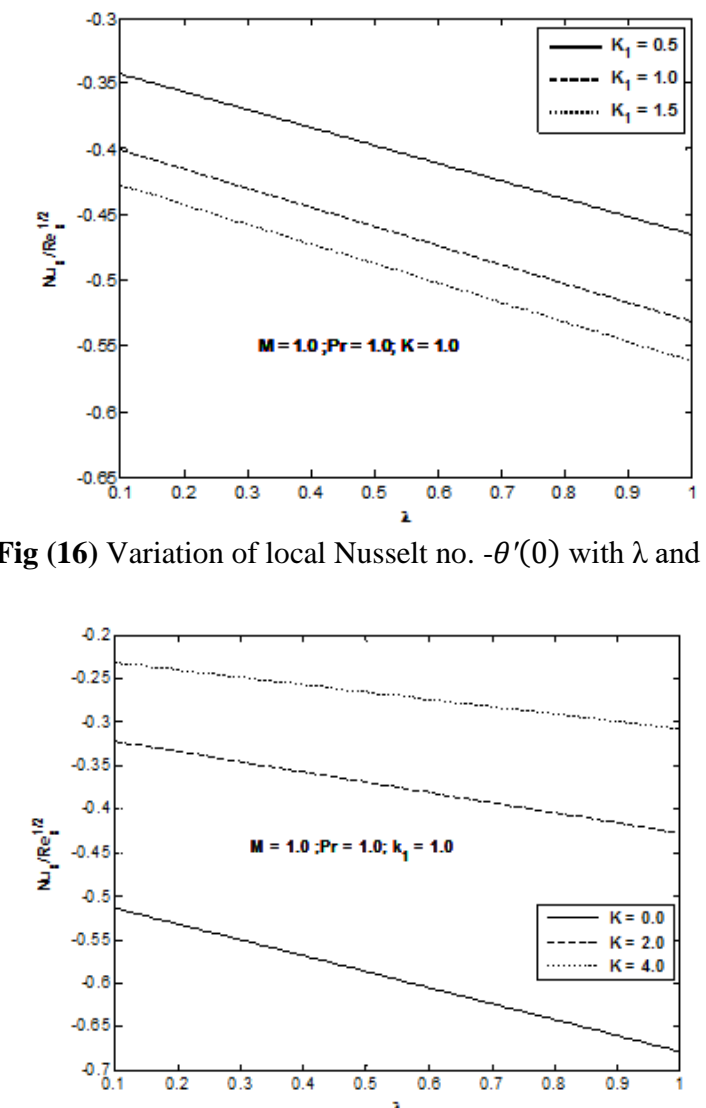

Fig (18) Variation of local Nusselt no. $-\theta^{\prime}(0)$ with $\lambda$ and $K$.
www.iosrjournals.org 


\section{Conclusions}

1. The velocity and angular velocity decreases with increase of material parameter whereas the temperature increases in both assisting and opposing flows.

2. The porous parameter and magnetic parameter enhances the velocity and angular velocity but decreases the temperature in both assisting and opposing flows.

3. With the increase of $\operatorname{Pr}$ the velocity and angular velocity decreases in assisting flow and increases in opposing flow while the temperature decreases in both assisting and opposing flows.

\section{References}

[1] A.C. Eringen, Theory of micropolar fluids, J.Math.Mech.16(1966), pp. 1-18.

[2] A.C. Eringen, Microcontinumm field theories. II: Fluent Media, Springer, Newyork, 2001.

[3] A.Ishak, R. Nazar, I. Pop, Moving vedge and flat plate in a micropolar fluid, Int.J.Eng.Sci.44(2006) 1225-1236.

[4] R.S.R.Gorla, Mixed convection in a micropolar fluid from a vertical surface with uniform heat flux, Int. J. Engi. Sci., 30, pp. 349-358, 1992.

[5] A.Ishak, R. Nazar, I. Pop, MHD flow of a micropolar fluid towards a stagnation point on a vertical surface, Computers and Mathematics with Applications 56(2008)3188-3194.

[6] Srinivasacharya. D, Ramana Murthy JV, Venugopalan D.Unsteady stokes flow of micropolar fluid between two parallel porous plates. Int J Eng Sci 2001; 39:1557-63.

[7] Bhargara R, Kumar L, Takhar HS. Numerical solution of free convection MHD micropolar fluid flow between two parallel porous vertical plates. Int J Eng Sci 2003;41:123-36.

[8] M.M. Rahman, M.A. Sattar, Transient convective flow of micropolar fluid past a continuously moving vertical porous plate in the presence of radiation, Int. J. App. Mech. Engi., 12(2), pp. 497-513, 2007. 